\title{
Improved calibration of color video projectors by means of computer animation
}

\author{
PIETER JAN STAPPERS \\ Delft University of Technology, Delf, The Netherlands
}

\begin{abstract}
A computer animation for the calibration of color video projectors is described. The animation facilitates the calibration procedure and can be readily programmed on almost any graphics computer or played from a videotape.
\end{abstract}

Color video projectors (CVPs) are widely used in applications requiring very large screen video images, such as presentations in large auditoriums or research settings in which a subject's visual field must be completely filled. CVPs (e.g., Barcodata PC and Sony VPH1030QM1) project image sizes of up to $3 \times 5 \mathrm{~m}^{2}$. In combination with advanced graphics computers, which are available at the low end of the market (e.g., the Amiga), they provide a powerful tool for displaying information-from simple line drawings to more complete simulated environments.

Unlike monitors, CVPs suffer the disadvantage of regularly needing calibration of the overlap of the red, green, and blue image-projecting tubes. If the overlap is not perfect, objects in the image will have colored edges. Furthermore, if the distance between CVP and projection screen has been changed, the lenses of all three tubes must be refocused. Many CVPs assist in these adjustments by generating an inbuilt calibration image, which consists of a grid of thin lines on a black background (Figure 1).

Calibration is a tedious process, sometimes requiring hours of control adjustments on the CVP while an attempt is made to adjust the thin lines of the grid for sharpness or overlap. These judgments are hard to make, because the operator working the controls is about $3 \mathrm{~m}$ away from the display screen, and the width of the lines in the calibration image subtend a visual angle of some seconds of arc.

Human observers are not very good at judging the sharpness of a low-intensity monochromatic line (needed for focusing a tube), or at judging the overlap of two such lines (needed for calibrating the overlap of the images of each pair of tubes). They are better at detecting changes than at judging details of static images. Furthermore, CVPs are typically used in a setting where such dynamic images as video films or computer animations are presented. (For static images, slide projectors yield a better image quality for a much lower price.) It therefore seems a waste not to use the dynamic power of the imagegenerating equipment. Instead of the operator having to judge the overlap of two vertical lines, a computer animation can ease the tuning by enhancing the visibility of

Requests for reprints may be sent to P. J. Stappers, Faculty of Industrial Design Engineering, Jaffalaan 9, 2628 BX Delft, The Netherlands.

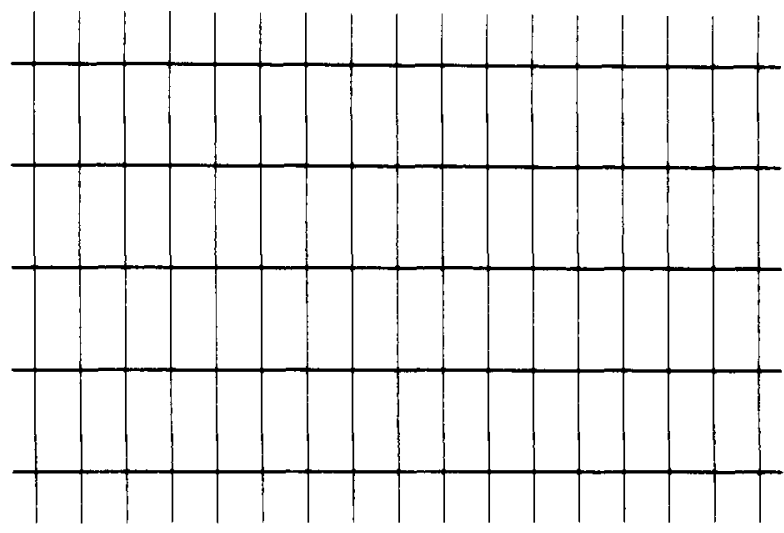

Figure 1. A conventional grid-shaped calibration image, as supplied by the Barcodata PC. In standard practice, this grid is shown separately for each tube to focus the lens and is projected simultaneously by two tubes to adjust overlap.

overlap errors. In the proposed procedure for adjusting horizontal overlap, for example, for the red and green images, the animation shows a vertical line, the upper half of which is red, the lower half green, on a yellow (red plus green) background (Figure 2).

Twice per second, the upper and lower halves exchange colors. If the red and green images are perfectly aligned, the red and green lines appear to jump up and down, but if the alignment is incomplete, the lines appear to jump sideways. The difference between jumping up and down versus jumping sideways is easier to judge than is the overlap of lines in a static image. In this way, overlap judgment depends on a combination of vernier and displacement judgments, acuity for which is much larger than that for separation, which is used in the static grid-overlay judgment (Olzak \& Thomas, 1986).

\section{Suggested Procedure}

Calibrating a CVP usually is a three-stage process. In the first stage, the projector tubes are focused separately. For this, the grid pattern or a pattern of large blocks, as in Figure 3, can be used.

In the second stage, one of the tubes, say, the green one, is adjusted to eliminate distortions (i.e., to ensure that straight lines appear straight and that circles do not 
become ellipses). This part still can be performed using a grid pattern, as in Figure 1. In the third stage, the red and blue images first are adjusted to overlap the green image. Rough-adjustment can best be done with the block pattern of Figure 3. For fine-tuning, the animation described above is used. Adjusting the overlap of red to green requires adjusting 10 to 20 controls. Use of the animation does not reduce the number of controls to be adjusted, but it does facilitate the adjusting of each of them. Each control causes a part of the picture to stretch, either horizontally or vertically. Its setting must be determined by judging overlap of the image at a point on the screen. It is at this point that the two lines in the animated picture should meet. If the control determines a horizontal stretch, the lines should be vertical, and vice versa. There are $\mathbf{2 0}$
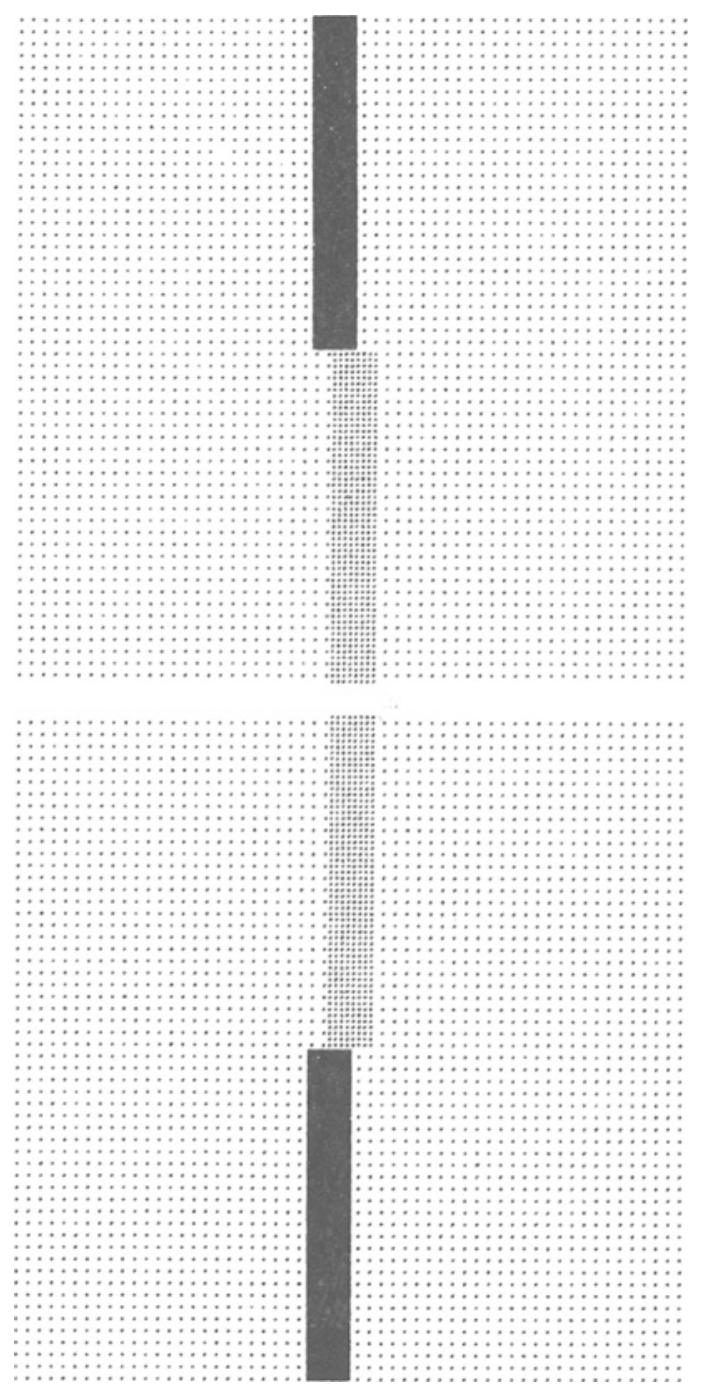

Figure 2. The line-pair animated image. Twice per second, the upper and lower lines exchange colors, leading to an apparent sideways motion if the color projection tubes are not aligned properly, an apparent up-and-down motion if they are. The block around the lines allows the operator to do rough-tuning, leaving the line pairs for fine-tuning.

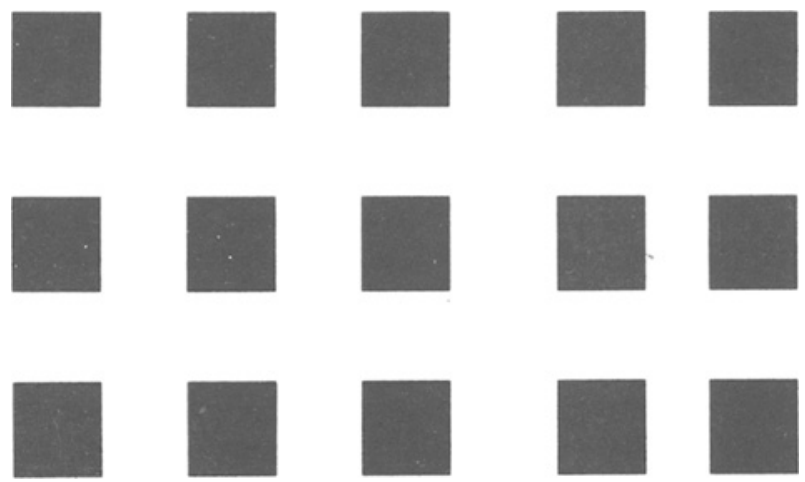

Figure 3. A simple pattern of large blocks for the focusing and rough-tuning of the overlap.

controls on, for example, a Barcodata PC, so there should be 20 animated line pairs. Showing all 20 pairs together results in a very confusing sight. Judgments are best made when only a few (preferably one) line pair(s) are presented simultaneously.

\section{Implementation}

A versatile solution is demonstrated by the computer program of Listing 1. The CVP receives the video or RGB output from the computer, and the operator can move an animated probe (as shown in Figure 2) about the screen by means of the computer mouse. In this way, the operator can place the probe in the location and orientation corresponding to the control he/she is currently calibrating.

The program first asks for the colors of the tubes that are to be adjusted to overlap. With, for example, the red and green tubes, a probe of a red and green line on a yellow (red plus green) background then is drawn and animated. When the operator clicks the mouse button, the probe is moved to the new mouse position and animated in another orientation (if it was vertical, it becomes horizontal, and vice versa). This continues until the program is stopped. The program can then be rerun for another combination of video tubes.

The program demonstrates the principle using standard screen sizes, which do not use the overscan region of the video signal. The program can be adapted for full video applications, using the system's graphics libraries (Commodore, 1986). Alternatively, the scheme also can be tried out in a matter of minutes by using a commercially available paint program (Silva, 1986) with overscan and colorcycling facilities. In fact, the animation was developed using such a paint program.

\section{REFERENCES}

Commodore Business Machines (1986). Amiga ROM Kernel reference manual: Libraries and devices. Reading, MA: Addison-Wesley.

OlzAK, L. A., \& ThOMAs, J. P. (1986). Seeing spatial patterns. In K. R. Boff, L. Kaufman, \& J. P. Thomas (Eds.), Handbook of perception and human performance, Vol. I. Sensory processes and perception. New York: Wiley.

Silva, D. (1986). DeluxePaint II. San Mateo, CA: Electronic Arts. 


\section{LISTING 1}

A Program Written in AmigaBasic, Demonstrating the Fine-Tuning Part of the Second Calibration Procedure Described in the Text

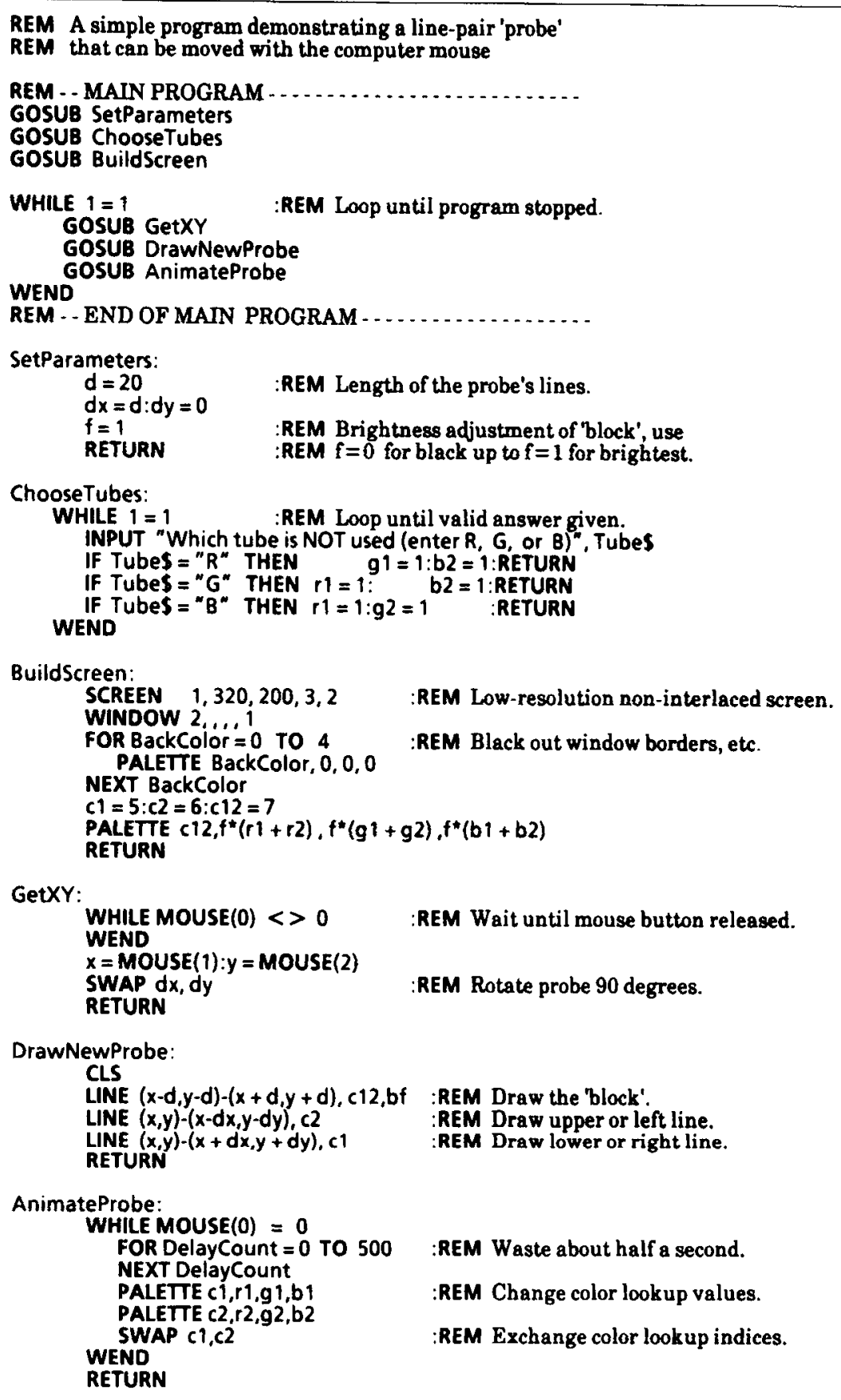

